\title{
Micro/Nano-Scale Optical Circuits and Networks: Issues, Challenges and Progresses
}

\author{
Prof. Dr. El-Hang Lee, Director \\ OPERA (Optics and Photonics Elite Research Academy) \\ National Research Center for Photonic Integration Technology \\ Micro/Nano-Photonics Advanced Research Center ( $\mu$-PARC) \\ Graduate School of Information and Communication Engineering, \\ INHA University, 253 YongHyun-Dong, Nam-Ku, Incheon, South Korea \\ www.opera.re.kr ehlee@inha.ac.kr
}

\begin{abstract}
$\underline{\text { Abstract }}$
We present a comprehensive overview of our original work on the theory, design, fabrication, and integration of micro/nano-scale optical circuits and networks $(\mathrm{O}-\mathrm{MNN})$. This is an extension of the macro-scale optical networks and electrical networks down to the level of micro/nano-scale. It also intends to compliment the limitations of the electrical circuits and networks by way of processing optical signals through micro/nano-optical networks while the electrical systems process electrical signals through the electrical networks. The micro/nano-optical circuits perform the functions of sensing, storing, transporting, processing, switching, routing, and distributing optical signals on flat modular boards or chips. The integrated optical components include micro/nano-scale light sources, waveguides, detectors, switches, modulators, sensors, directional couplers, multi-mode interference devices, AWGs, ring-resonators, photonic crystal devices, plasmonic devices, and quantum devices, made of polymer, silicon and other semiconductor materials. Some molecular devices are also considered. The paper discusses scientific and technological issues and approaches concerning the miniaturization, interconnection and integration of micro/nano-scale photonic devices, circuits, and networks leading to ultra-small and very large scale integration as applicable for datacom, telecom, transportation, aero/space/avionic and bio/sensor/environmental systems. Scaling rules for the miniaturization and integration of the micro/nano-photonic systems will be discussed in comparison with those of the electronic systems. New physics, visions and challenges of the optical micro/nano-optical networks and systems will be discussed along with the historical perspectives of the electrical technology. Progresses and examples are presented.
\end{abstract}

\section{INTRODUCTION}

In the historical perspective, electrical networks based on copper wires and copper cables dominated world over the many decades from the early $20^{\text {th }}$ century through the $1970 \mathrm{~s}$. The electrical wires and networks continued their penetration down to the level of centimeter and millimeter ranges by way of what we know of today as electrical printed circuit boards and to the micro/nano-scale regime that we now know as VLSI integrated circuits. Then, the optical fibers and optical cables arrived to replace the copper cables and electrical networks to build optical networks and optical internets that we know of and use today. But, much of the optical networks exist only in the macro-scale dimensions from tens of thousands kilometers down to tens of meters. There is still much room to explore in terms of optical networks down to the centimeter, millimeter, micron and nano-scale domain. [1-9]

Here, in an extended effort, we carry this concept to the domain of optics and photonics and perform a systematic study on the micro/nano-scale optical networks toward VLSI photonic circuits and networks. It is also an extension of the macro-scale optical fiber network that has revolutionized the world of telecommunication into the domain of micro/nano-scale network. [1-9]

This paper presents an overview of the collective original work on the theory, design, fabrication, and integration of the micro/nano-scale optical networks. Micro/nano-scale photonic wires and devices are interconnected on a planar board or on a chip to form a network of various functions that are compact, high- 
speed, intelligent, light-weight, low-energy and environmentally friendly, low-cost, and high-volume applications. Photonic devices include micro/nano-scale lasers, switches, couplers, detectors, sensors, actuators, modulators, and related devices.

Applications of the optical micro/nano-networks can vary from telecom applications to the datacom applications, automobile applications, bio-sensor applications, medical applications and environmental applications, and aero-space applications.

\section{SCIENTIFIC ISSUES IN NANO-ELECTRONIC CIRCUITS AND NETWORKS}

Size Effect: Issues of spatial quantization, quantum confinement effect, quantum resonance effect, quantum interference effect, and the image force emerge as important issues.

High Field Transport Effect: Electron transport through small channels and areas can induce effects like velocity overshoot, ballistic transport, hot electron effect, and hot phonon effect. Problems of anisotropic diffusion, oxide polar phonons, and decreasing channel mobility can also arise.

Environmental Effect: This aspect becomes important to devices of small sizes. These include low-level radiation effetcs and synergetic effects.

Generation-Recombination Effect: Hot electron thermionic emission and impact ionization can arise as important issues when the sizes of the electronic devices become small.

\section{SCIENTIFIC ISSUES IN NANO-PHOTONIC CIRCUITS AND NETWORKS}

Size Effect: As the sizes of devices become small, the proximity effect, the energy confinement effect, the microcavitiy effect in microlasers, single photon effect, and the optical interference effect between devices become important issues. The issues in microelectronics, mentioned above, will also emerge as important issues in micro/nano-photonics, especially in the devices where the role of electrons is significant for the generation and control of photons. Solid state theories for bulk devices may need to be refined. Nonlinear effects can become pronounced. Interface physics and environmental issues also become important.

High-Field Nonlinear Effect: The high field intensity of the light within the devices can cause nonlinear interactions. Nonlinear effects can be useful for new functional devices but they can also degrade the device performances. Hot electron effects under high field can induce hot phonon effects and impact ionization. These issues need special attention when nano-photonic devices are integrated with nano-electronic devices.

Noise Effect: Understanding and controlling the dynamics of noise in small photonic devices becomes important. Noises may include quantum optical and chaotic noises.

Quantum-Optical Effect: Quantum chaotic phenomena and quantum interference effect become important. Identifying the semi-classical limit is important. Quantum optics is expected to become an important part of micro/nano-photonics study.

\section{SCALING DESIGN RULES}

\subsection{Scaling in Micro/Nano-Electronics}

In micro/nano-electronics, device sizes are reduced with certain guidelines or rules so that all the parameters are scaled down without losing balanced performance of the devices. These rules or laws are called «scaling rules» or «scaling laws», which can vary depending on what parameter is fixed. The parameters to be scaled include the vertical and surface dimensions, the circuit time, the currents, voltages, power density, and power dissipation. Scaling law is essential in the design of micro/nano-electronic devices.

\subsection{Scaling in Micro/Nano-Photonics}

In the design of micro/nano-photonic devices, however, no scaling rules or laws have been yet established to provide guidelines for miniaturization or integration. There are sporadic reports on the effect of scaling on the 
performances of the photonic devices, but the scaling laws for photonic device should be more systematically established. Parameters to be put into consideration, among others, should include: device dimension, size, electrical current, light output power, input power, wavelength, refractive index, mode, polarization, and device proximity, in addition to all the effects discussed above. This is certainly an area of challenge to be addressed in the years to come. We have been able to come up with preliminary scaling rules both for miniaturization and integration that we have been able to use for the design of micro/nano-photonic directional couplers.

\section{DENSITY CALCULATIONS FOR MICRO-SCALE WAVEGUIDE INTEGRATION}

We identified some design rules for the minimization of the waveguide sizes and the maximization of the integration density of the optical wires by way of theoretical and simulation studies. For a crosstalk of $-30 \mathrm{~dB}$ for a family of different waveguide with refractive index difference of 0.02 , for example, the minimum waveguide width and the optimum separation between waveguides comes out to be $7 \mu \mathrm{m}$ and $14 \mu \mathrm{m}$, respectively, allowing for 46 waveguides within a $1 \mathrm{~mm} \times 100 \mathrm{~mm}$ substrate.

\section{BANDWIDTH CALCULATION FOR MICRO/NANO-NETWORKS}

We also calculate the bandwidths for various sizes of waveguide width and for cross sections varying from 1 micron to 150 microns with index difference as 0.2 , for the refractive indices of the core and the cladding fixed for 1.47 and 1.45. For an O-PCB with 100 micron width and 100 micron height, for example, the bandwidth is $62 \mathrm{Gbps}$ for $10 \mathrm{~cm}$ length. As the cross-sectional area diminishes, the bandwidth increases exponentially, even up to several Tera Gbps range. For copper, the theoretical limitation of the bandwidth is less than 60Gbps.

\section{MICRO/NANO-PHOTONIC COMPONENTS AND CIRCUIT INTEGRATION}

We design and integrate arrayed waveguide devices, directional couplers, multi-mode interference devices, micro-ring resonant devices with the polymer optical wires that are pre-wired and pre-deposited on the OPCB board. We use mode adapters for the integration of these devices. And we also examine the compatibility of interconnection and integration between many different devices: between the round-shaped optical glass fibers with the polymer waveguide of rectangle-cross-section; between the waveguides and the photonic crystal waveguides; between the waveguides and the plasmonic waveguides; between the plasmonic waveguides with the quantum structures, for example. The scientific and technical issues and challenges for these interconnections and integrations include the size mismatch, the polarization mismatch, the thermal mismatch, the mechanical mismatch, and the shape mismatch

\section{SILICON-BASED MICRO/NANO-PHOTONIC CIRCUITS AND NETWORKS}

We design and integrate silicon based micro-ring resonant devices and photonic circuits for sensor applications. We measure the changes in the refractive indices of the molecules and/or bio-materials coming in to touch with the micro-ring surfaces. We also design and fabricate silicon based nano-wires and photonic crystal devices and circuits for nano-scale optical interconnection and integration.

We also design silicon microstructures in which we can confine the light between two waveguides. We then extend this to a multiple array of such waveguide pairs for confinement of multiple beams of light. Silicon light amplifiers are also designed and have been demonstrated using rare earth dopants.

\section{PHOTONIC CRYSTAL MICRO/NANO-CIRCUITS AND NETWORKS}

Photonic crystals, where refractive index is periodically modulated to photonic dispersion and photonic band gap analogous to the electronic band gap in a semiconductor, can be utilized for ultra-small optical devices 
such as waveguides, add/drop filters, switches, modulators, and high efficient cavities for VLSI photonic integrated circuits.

To realize nano-scale VLSI optical circuits, we have designed power-splitting devices using 2-dimensional photonic crystals. In order to increase the density of integration of the waveguides, we used parallel-shaped directional couplers in photonic crystals, and have found this structure superior to other structures, such as Ybranch (or junction) 2-dimensional photonic crystal power splitters, which seem to take up too much space for VLSI nano-photonic circuits.

In another study of photonic crystal for VLSI photonic circuit application, we employed self-collimating phenomena and super-prism phenomena to design a polymeric photonic crystal wavelength-splitter. We fabricated a polymeric photonic crystal wavelength-splitter using ultra-violet (UV) embossing technique that can be simply performed at room temperature with a silicon mold. In a 3-dimensional finite difference timedomain (3-D FDTD) simulation, the light beams of two different wavelengths (1043-nm and 1550-nm) are separated at an angle of 15 degrees in the photonic crystal region. The two light beams with different wavelength, however, are not separated without photonic crystal structure. We expect that this design concept would enable one to realize very high-density integrated circuits due to its simple fabrication process and compact size.

We also designed a two-dimensional passive optical network triplexer by using the photonic crystal. By varying the sizes and the refractive indices of the localized defects in the photonic crystal, we have been able to generate curves that can determine the transmission wavelengths. Here, we used a two-dimensional photonic crystal with a square pattern of rods and have been able to use three kinds of lights with free space wavelengths, $\lambda_{1}=1550 \mathrm{~nm}, \lambda_{2}=1490 \mathrm{~nm}$, and $\lambda_{3}=1310 \mathrm{~nm}$ for the multiplexing. We set the lattice constant, $a=490 \mathrm{~nm}$, and obtained the corresponding frequencies and radii for each wavelength by using the FDTD calculation.

For VLSI optical networks and circuits, we use photonic crystals. We design, for example, a two-dimensional passive optical network (PON) triplexer by using the photonic crystal. Since the frequencies of defect modes can be varied with the radii of defects, one can control the coupling frequencies of defect modes. The tuning of the transmitted wavelength can be done by way of controlling the size of the air holes or by changing the values of the refractive indices of the photonic crystals.

We also employ self-collimating phenomena and super-prism effect to design and fabricate a polymeric photonic crystal wavelength-splitters, filters, and power splitters. We expect that this design concept and lowcost mass production method of a photonic crystal based device enables us to realize a very high-density integrated circuits due to its simple fabrication process and compact size.

\section{PLASMONIC MICRO/NANO-CIRCUITS AND NETWORKS}

We also use surface plasmon-polaritons (SPPs) formed on a flat metal surface like silver or gold for micro/nano-photonic circuits and networks. In our study, the stripe waveguides were formed by using $20 \mathrm{~nm}$ thin and 5um wide gold strips sandwiched between $12 \mathrm{um}$ thick spin coated layer of polymer supported by silicon wafer. Fundamental SPP mode, excited by end-fire coupling at telecommunication wavelengths (1.52 1.58um) with polarization controlled (PC) single mode fiber, for example, showed the guiding propagation loss of $\sim 19 \mathrm{~dB} / \mathrm{cm}$ and the coupling loss with the fiber of about $\sim 1.8 \mathrm{~dB}$ per facet. Vertical directional couplers (VDCs) of $20 \mathrm{~nm}$ thin and $5 \mathrm{um}$ wide gold stripes, consisting of metal stripe waveguide embedded in polymer, revealed the extinction ratio of about $28 \mathrm{~dB}$ and the separation distance of about $4.2 \mathrm{um}$, indicating a possibility of high density integration. For a strip separation of $4.2 \mathrm{um}$, the power transfer was as much as $\sim 28 \mathrm{~dB}$ with the interaction length of $260 \mathrm{um}$ and the total insertion loss was about $\sim 24 \mathrm{~dB}$. 


\section{ASSEMBLY OF THE MICRO/NANO-NETWORKS}

The networks were assembled with an array of $1 \times 12$ polymer waveguides with the built-in $45^{\circ}$ ended mirrors fabricated by UV embossing. The refractive index of the waveguide is 1.47 at $850 \mathrm{~nm}$. The dimensions of the waveguides are 50 micron in width and height with a pitch of 250 micron. Arrays of vertical cavity surface emitting lasers (VCSELs) and photodiodes (PDs) are wire-bonded or flip-chip bonded to the silicon devices for optical input-output $(\mathrm{I} / \mathrm{O})$ interconnection. When the light emitted from the VCSEL was set to $+3 \mathrm{dBm}$, the fiber's light receiving power through the waveguide was found to be $-4.9 \mathrm{~dB}$ and the total loss, including the propagation loss and coupling loss between optical transmitter and waveguide, was $-7.9 \mathrm{~dB}$. We have been able to measure the transmission speed of up to $10 \mathrm{Gps}$ for each channel using the eye diagram. We also have been able to fabricate the circuits on hard and flexible substrates and have been able to measure the speed up to 10Gbps. Micro/nano-circuits and networks are then interconnected and integrated using these arrays of waveguides.

\section{PERFORMANCE TESTS OF THE MICRO/NANO-NET VLSI NETWORKS}

We tested the assembled micro-scale by watching a moving video stored in a memory chip by interconnecting the memory chips and the CPU chips using optical wires. What we have done is to cut the copper wires connecting the memory chip and the CPU chip and replace all the copper wires with the optical wires. We used the electrical signal from the memory device to provide the optical signals from a semiconductor laser (VCSEL, for example) and guided these optical signals from the VCSEL laser to the detectors by way of polymer optical wires. We have been able to watch the moving images through the optical wires.

\section{SUMMARY AND CONCLUSION}

We presented an overview of the new challenges that the OPERA National Research Center for Photonic Integration Technology is investigating on the theory, design, fabrication, and integration of micro/nano-scale optical circuits and networks (O-MNN) toward "VLSI photonic integrated circuits"(VLSI-PICs). Theoretical, experimental and simulation studies suggest that the micro/nano-scale network is a new challenge and warrants much study.

\section{ACKNOWLEDGEMENTS}

This work has been supported by the Korea Science and Engineering Foundation (KOSEF) through the Grant for the Integrated Photonics Technology Research Center (R11-2003-022) at the Optics and Photonics Elite Research Academy (OPERA), Inha University, Incheon, South Korea.

\section{REFERENCES}

1. E. H. Lee, Invited Talk, SPIE Photonics West, San Jose, CA, USA. Jan. 22-26, 2007

2. E. H. Lee, Keynote Lecture, "Optical Micro/Nano-Networks" IASTED2006, July 2-4, 2006, Banff, CANADA

3. E. H. Lee, Plenary Lecture, "VLSI Photonics," ICSEP2006, April 1-4, 2006, Taipei, TAIWAN

4. E. H. Lee, Invited Lecture, SPIE-Europe, April 3-7, 2006, Strasbourg, FRANCE

5. E. H. Lee, Invited Talk, SPIE Photonics West, Proceedings, Vol. 5729, pp. 118, 2005

6. E. H. Lee, Invited Talk, SPIE Photonics West, Proceedings, Vol. 4652, 2002, p.1.

7. E. H. Lee, Invited Talk, SPIE Photonics West, Proceedings, Vol. 5356, 2004

8. E. H. Lee, Invited Talk, IEEE/LEOS, Summer Topical Meeting, June 29-30, 2004, San Diego, CA, USA.

9. Inha University, (2005) Integrated Photonics Technology Research Center, Optics and Photonics Elite Research Academy (OPERA), Home Page Address: http://www.opera.re.kr 